\title{
Une chance pour la médecine de la transplantation suisse
}

Franz F. Immer

Dr méd., médecin spécialisé en chirurgie cardiaque et directeur de Swisstransplant, membre FMH

Cette année, la Suisse est le pays organisateur de la Journée européenne du don d'organes et de la transplantation (EODD). Le 9 septembre, Swisstransplant célébrera cet événement sur la Place fédérale avec des donneurs et des receveurs, l'Office fédéral de la santé publique (OFSP), des représentants du monde politique et des personnalités de la vie publique. Cette journée nous donne la possibilité d'échanger au niveau international avec des experts du domaine du don d'organes et de la greffe et, surtout, de sensibiliser la population au sujet.

Depuis 1996, le Conseil de l'Europe et un pays donné organisent tous les ans la Journée européenne du don d'organes et de la transplantation (European Day for Organ Donation and Transplantation, EODD) afin de sensibiliser l'opinion publique. Cette journée s'inscrit dans le cadre des efforts communs de la Commission européenne, du Conseil de l'Europe et des États membres afin d'augmenter le nombre de donneurs, de soutenir la médecine de la transplantation, et donc d'améliorer les transplantations en termes de qualité et de sécurité. En 2017, c'est la Suisse qui accueille l'EODD. Une cérémonie officielle sera célébrée parallè- lement à Genève pour la Journée mondiale du don d'organes et de la greffe (World Day of Organ Donation and Transplantation, WDODT). Et la Journée nationale du don d'organes aura lieu aussi à cette date. Pour la première fois, ces trois événements se tiendront le même jour, dans le même pays.

\section{Le rôle de pionnier de la Suisse}

Swisstransplant considère l'attribution de l'EODD 2017 à la Suisse comme un signe de reconnaissance pour le rôle joué par le pays dans l'histoire de la médecine de la

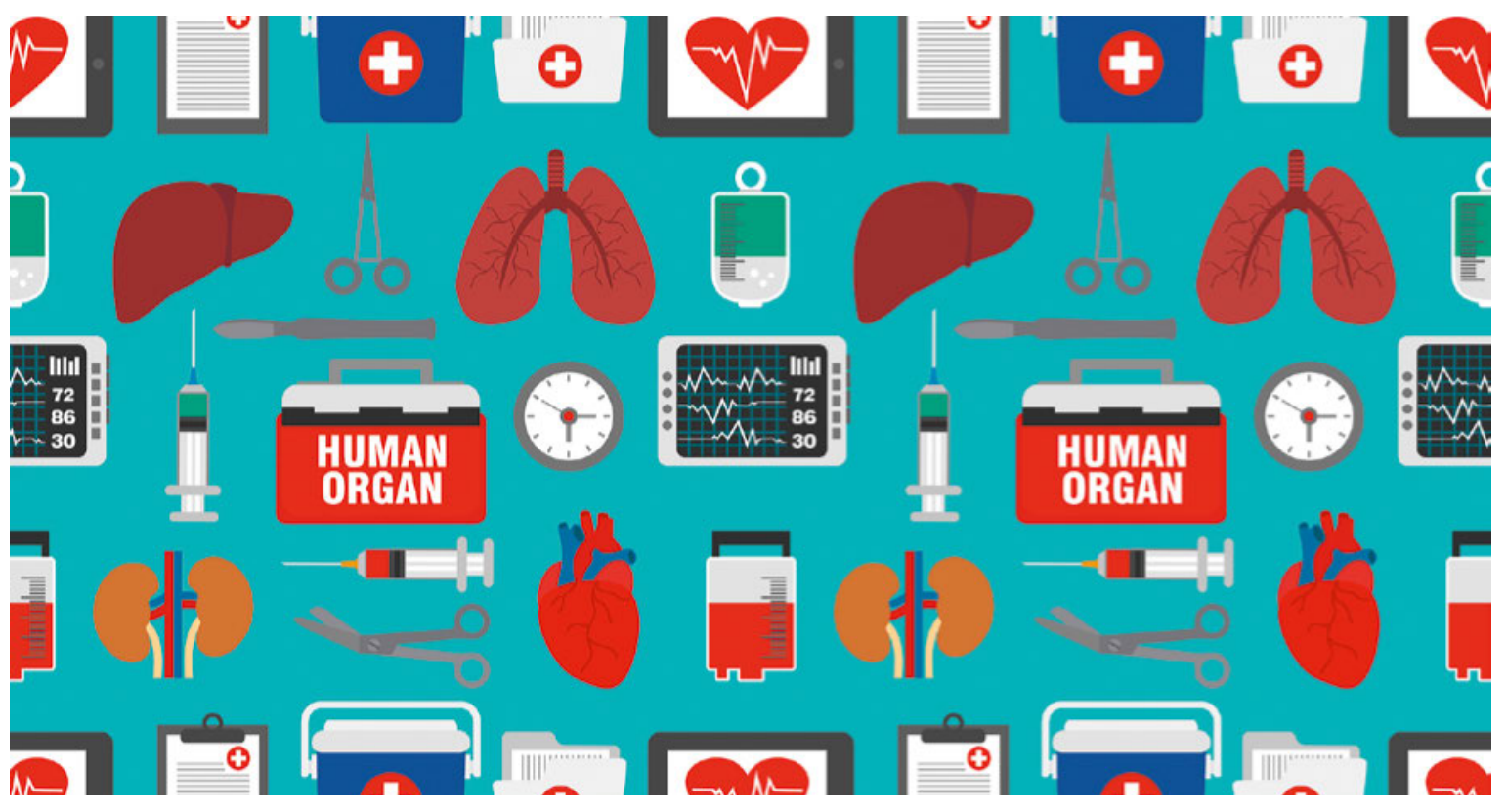


transplantation, qui a commencé en 1883. Le chirurgien bernois Theodor Kocher (1841-1917), qui recevra plus tard le prix Nobel, greffe du tissu thyroïdien d'origine humaine sous la peau et dans la cavité abdominale d'un jeune homme ayant subi une ablation de la thyroïde. Il s'agit d'une véritable percée pour l'époque et du début de la thérapie de transplantation. Un autre jalon est posé avec la découverte de la ciclosporine par Hartmann Stähelin et Jean-François Borel chez Sandoz à Bâle, au début des années 70. Les premiers immunosuppresseurs, qui empêchent le rejet des organes greffés, ont été développés sur ces bases. Depuis cette époque, la Suisse n'a cessé d'apporter sa contribution à l'évolution de la médecine de la transplantation. C'est un honneur pour nous d'organiser l'EODD 2017. Nous sommes impatients de vous accueillir et de pouvoir échanger avec de nombreux spécialistes européens du domaine de don d'organes et de la transplantation.

\section{L'idée de l'EODD}

L'idée qui se cache derrière cette journée est d'aider tous les ans un état membre à sensibiliser la conscience publique et à susciter des débats sur le don d'organes et la transplantation. En raison de la pénurie d'organes transplantables, la liste d'attente ne s'allonge pas seulement en Suisse, mais aussi dans toute l'Europe. Les besoins d'organes ne cessent en outre d'augmenter. Swisstransplant aimerait informer correctement chacun afin de l'inciter à se pencher sur le don d'organes, à se décider et à faire part de sa décision à ses proches. Les hôpitaux et les spécialistes nous soutiendront aussi en cette journée dans l'espoir de mobiliser un plus grand nombre de donneurs potentiels. Ce jour de commémoration est également l'occasion de rendre hommage à tous les donneurs et à leur familles et de remercier les spécialistes du don d'organes et de la transplantation dans toute l'Europe. Leur travail permet en effet de sauver des vies et d'améliorer la qualité de celle d'une multitude de personnes.

\section{Des visages et des histoires}

Swisstransplant veut profiter de l'EODD pour sensibiliser l'opinion au don d'organes grâce à une campagne sur les réseaux sociaux, pour se confronter aux préjugés et corriger les fausses opinions existantes. Six mini-documentaires de deux à trois minutes brossent le portrait de personnalités du don d'organes et de la transplantation, à savoir des responsables médicaux des réseaux de don d'organes suisses, des donneurs potentiels, une chirurgienne de la transplantation, des receveurs et des proches de donneurs. Vous y découvrirez, par exemple, les deux amis si différents que sont Matthias et Gianni. Leur destin commun de receveurs leur a permis de se rencontrer et ils jouent aujourd'hui ensemble à la pétanque pour se préparer aux Jeux Mondiaux des Transplantés. Ou encore Michelle, une femme débordant d'énergie, que personne ne prendrait pour une transplantée du cœur. Baavalan, lui, a été transplanté du foie dans son enfance. Il est maintenant étudiant en médecine et postule à Genève pour un stage auprès du médecin qui l'a opéré à l'époque. Citons aussi Sébastien, le moniteur de conduite et pilote de course qui, conscient de la dangerosité de son loisir et motivé par un cas de maladie dans sa famille, a dit «Oui» au don d'organes. Et pour finir Barbara, qui a perdu son mari et a dû, dans cette difficile situation, se décider seule en faveur du don d'organes, car le sujet n'avait jamais été abordé auparavant dans la famille.

Ces histoires bouleversantes montrent à quel point il est important de parler du don d'organes et de se décider pour décharger ses proches. Vous trouverez ces films et d'autres informations sur l'EODD sur www.eodd2017.ch.

Nous vous attendons avec impatience sur la Place fédéral de Berne.

\section{Crédit photo}

(c) Dicraftsman | Dreamstime.com 